\title{
Низкоразмерные структуры карбида кремния: аналитические оценки характеристик электронного спектра
}

\author{
(C) С.Ю. Давыдов \\ Физико-технический институт им. А.Ф. Иоффре Российской академии наук, \\ 194021 Санкт-Петербург, Россия \\ E-mail: Sergei_Davydov@mail.ru \\ Поступила в Редакцию 30 сентября 2019 г. \\ В окончательной редакции 9 января 2020 г. \\ Принята к публикации 14 января 2020 г.
}

\begin{abstract}
Методом функций Грина в представлении сильной связи рассмотрена электронная структура бесконечного листа карбида кремния и вырезанных из него нанолент и одномерных цепочек. Получены аналитические выражения для ширин запрещенных зон, эффективных масс и характерных скоростей электронов. Обсуждается влияние металлических и диэлектрических подложек на зонные характеристики.
\end{abstract}

Ключевые слова: зонная структура, эффективная масса, характерная скорость, наноленты, цепочки, подложка.

DOI: $10.21883 / F T P .2020 .05 .49257 .9274$

\section{1. Введение}

После выхода на авансцену графена $[1,2]$, представляющего чрезвычайный интерес как с фундаментальной $[3,4]$, так и с прикладной $[5,6]$ точек зрения, начался поиск (главным образом, теоретический) других двумерных (2D) структур, обладающих, по возможности, преимуществами графена и лишенных его недостатков. К недостаткам следует отнести отсутствие щели (запрещенной зоны) в электронном спектре идеального однолистного графена, находящегося в свободном состоянии, что ограничивает возможности его использования в приборных структурах. Для возникновения такой щели приходится прибегать к так называемой функционализации графена, в качестве которой могут выступать допирование примесями, чужеродными адсорбированными атомами и подложками, а также неоднородная деформация листа графена. Такой функционализации не требуется, если 2D структура изначально содержит щель.

В достаточно длинном ряду выявленных за последнее десятилетие графеноподобных соединений (ГПС) [7-12] несомненный интерес представляют 2D аналоги бинарных полупроводников $A_{N} B_{8-N}[10,13-18]$, среди которых наиболее изучен гексагональный нитрид бора. В настоящей работе будут рассмотрены свойства $2 \mathrm{D} \mathrm{SiC}$ как одного из потенциально перспективных представителей семейства ГПС. Мнение о перспективности 2D $\mathrm{SiC}$ основано на свойствах объемного карбида кремния, характеризуемого, с одной стороны, высокой механической прочностью, а также тепло- и радиационной стойкостью (что роднит его с графеном), а с другой - являющегося широкозонным полупроводником $[19,20]$. Помимо бесконечного листа, будут рассмотрены также наноленты и цепочки карбида кремния. При этом основное внимание уделяется главным характеристикам зонного спектра, а именно ширинам запрещенных зон, скоростям Ферми, эффективным массам носителей в свободных структуpax, a для эпитаксиальных наноструктур - влиянию субстратов (металлического и диэлектрического) на эти характеристики. Отметим, что все полученные далее формулы справедливы для любых бинарных 2D соединений.

\section{2. Плоский лист карбида кремния}

В первой, по-видимому, работе по 2D $\mathrm{SiC}$ [21] для бесконечного листа двумя различными методами были вычислены значения ширины прямой запрещенной зоны $E_{g}$ (щели), равные 2.58 и 3.94 эВ. Расчеты $E_{g}$ были продолжены в работах [9,13-15,22-24], результаты которых приведены в табл. 1. Было показано также, что структура 2D SiC является плоской. Подчеркнем, что все цитированные работы представляют собой численные расчеты, выполненные в рамках DFT (density functional theory) для свободного $\mathrm{SiC}$-листа.

В $[25,26]$ нами была предложена простая модель описания свойств ГПС, находящихся как в свободном состоянии, так и сформированных на металлах [25] и полупроводниках [26]. В свободном состоянии энергия дисперсии $\pi$-электронов в ГПС имеет вид

$$
\begin{gathered}
E_{ \pm}(\mathbf{k})=\bar{\varepsilon} \pm R(\mathbf{k}), \\
R(\mathbf{k})=\sqrt{\Delta^{2}+t^{2} f^{2}(\mathbf{k}) .}
\end{gathered}
$$

Здесь

$$
f(\mathbf{k})=\sqrt{3+2 \cos \left(k_{x} a \sqrt{3}\right)+4 \cos \left(k_{x} a \sqrt{3} / 2\right) \cos \left(3 k_{y} a / 2\right)},
$$

где $\mathbf{k}=\left(k_{x}, k_{y}\right)$ - волновой вектор для движения электрона в плоскости листа, $a-$ расстояние между ближайшими соседями; $\bar{\varepsilon}=\left(\varepsilon_{A}+\varepsilon_{B}\right) / 2$, $\Delta=\left(\varepsilon_{A}-\varepsilon_{B}\right) / 2$, где $\varepsilon_{A(B)}$ - энергия $p$-орбитали атома $A(B) ; \quad E_{+}(\mathbf{k}) \quad$ - отвечает зоне проводимости, $E_{-}(\mathbf{k})$ - валентной зоне. Ширина щели в спектре 
Таблица 1. Расчетные значения ширины запрещенной зоны для бесконечного листа карбида кремния

\begin{tabular}{l|c}
\hline \multicolumn{1}{c|}{$E_{g}$, эВ } & Ссылка \\
\hline $2.58,3.94(\mathrm{KK})$ & {$[21]$} \\
$2.55(\mathrm{KK})$ & {$[22]$} \\
$2.52,4.19$ & {$[13]$} \\
$3.53(\mathrm{KK})$ & {$[14]$} \\
$2.55(\mathrm{KM})$ & {$[9]$} \\
$2.34,2.39(\mathrm{KM})$ & {$[15]$} \\
$2.53(\mathrm{KK})$ & {$[23]$} \\
$2.58(\mathrm{KK})$ & {$[24]$} \\
$2.45,3.48($ КК $)$ & Настоящая работа
\end{tabular}

Примечание. В скобках первый и второй символы обозначают точки зоны Бриллюэна 2D SiC, отвечающие соответственно краям валентной зоны и зоны проводимости.

при $\mathbf{q}=\mathbf{K}-\mathbf{k}=0$, где $\mathbf{K}=a^{-1}(2 \pi / 3 \sqrt{3}, 2 \pi / 3)-$ волновой вектор точки Дирака, равна $E_{g}=2 \Delta$. Используя таблицы атомных термов Хермана-Скиллмана [27] $\left(E_{\mathrm{C}}=-8.97\right.$ эВ, $\varepsilon_{\mathrm{Si}}=-6.52$ эВ относительно вакуума $)$ и Манна [28] $\left(\varepsilon_{\mathrm{C}}=-11.07\right.$ эВ, $\varepsilon_{\mathrm{Si}}=-7.59$ эВ), получим соответственно $E_{g}=2.45$ и 3.48 эВ [25], что вполне удовлетворительно согласуется с приведенными в табл. 1 величинами. Интересно отметить также, что разброс расчетных значений $E_{g}$ практически совпадает с интервалом ширин запрещенных зон для известных 3D-политипов $\mathrm{SiC}: E_{g}$ равно 2.40 эВ для 3С-политипа и 3.33 эВ для $2 H$-политипа [29].

В свободном листе ГПС эффективные массы электронов $(e)$ и дырок $(h)$, определяемые стандартным выражением $m_{e, h}^{-1}= \pm \hbar^{-2}\left(\partial^{2} R(\mathbf{q}) / \partial q^{2}\right)_{q=0}(\hbar-$ приведенная постоянная Планка), удобно представить в виде

$$
\frac{1}{m_{e}}=\frac{c^{2}}{\Delta}
$$

где $c=3 a t / 2 \hbar-$ параметр с размерностью скорости (аналог скорости Ферми в графене), для 2D $\mathrm{SiC}$ $a=1.77 \AA[25]$ и $t=1.53$ эВ - энергия перехода электрона между ближайшими соседями (б.с.), рассчитанная по теории Харрисона [28]. Отсюда получаем значения $m^{*} \equiv m_{e} / m_{0}=-m_{h} / m_{0}\left(m_{0}-\right.$ масса свободного электрона), равные 0.57 и 0.81 при использовании соответственно таблиц [27] и [28]. Для сравнения: в объемных образцах карбида кремния $m^{*}=0.44$ для $3 C-\mathrm{SiC}$ и $m^{*}=0.71$ для $2 \mathrm{H}$-SiC [29], что достаточно близко к найденным нами значениям. Для $2 \mathrm{D} \mathrm{SiC}$ численный расчет [9] дает $m_{e} / m_{0}=0.41$ и $\left|m_{h}\right| / m_{0}=0.49$, что достаточно удовлетворительно согласуется с нашими результатами, полученными с использованием таблиц Хермана-Скиллмана.

Перейдем теперь к эпитаксиальному монослою карбида кремния и начнем со случая металлической подложки, рассмотренного в [25]. Пусть плотность состояний металлического субстрата $\rho_{m}(\omega)=\mathrm{const}$, где $\omega$ - энергетическая переменная, и уровень Ферми системы $E_{\mathrm{F}}$, диктуемый подложкой, лежит вблизи центра запрещенной зоны ГПС $\left(\left|E_{\mathrm{F}}-\bar{\varepsilon}\right| \ll t\right)$. Тогда для среднего числа заполнения $\bar{n}=1+v(|v| \ll 1)$ одного атома элементарной поверхностной ячейки получим следующее выражение:

$$
v \approx \frac{2 \Gamma_{m} E_{\mathrm{F}}}{\pi^{2} \sqrt{3} t^{2}} \ln (\sqrt{2 \pi \sqrt{3}} t / \Delta),
$$

где $\Gamma_{m}=\pi \rho_{m} V^{2}$ - уширение уровней карбида кремния, вызванное взаимодействием с подложкой $V$ [30]. Концентрация носителей в ГПС равна $N_{c}=v / S_{1}$, где $S_{1}=3 \sqrt{3} a^{2} / 4$ - площадь, приходящаяся на один атом элементарной ячейки ГПС. Отметим, что при $E_{\mathrm{F}}>0$ носителями тока являются электроны, при $E_{\mathrm{F}}<0-$ дырки.

Оценим теперь, как металлическая подложка влияет на эффективную массу носителей. Воспользовавшись результатами работы [31], в общем случае имеем

$$
m^{*} / \widetilde{m}^{*}=F(\bar{\varepsilon}), \quad F(\omega)=(1-d \lambda(\omega) / d \omega)^{-1},
$$

где $\widetilde{m}^{*}$ - эффективная масса в присутствии подложки, $\Lambda(\omega)$ - функция сдвига энергетических уровней ГПС [30] вследствие взаимодействия с подложкой. Так как плотность состояний металла $\rho_{m}=$ const, имеем $\Lambda_{m}(\omega)=0$ [30], так что $m^{*} / \widetilde{m}^{*}=1$.

Пусть теперь подложка представляет собой диэлектрик: например, $\mathrm{BN}$ с запрещенной зоной $E_{g}^{\text {sub }} \sim 6$ эВ или $\mathrm{SiO}_{2}$ с $E_{g}^{\text {sub }} \sim 9$ эВ. Как и в $[26,31]$, зададим плотность состояний подложки в виде $\rho_{\text {diel }}(\omega)=$ $=A \sqrt{|\omega|-E_{g}^{\text {sub }} / 2}$ при $|\omega|>E_{g}^{\text {sub }} / 2$ и $\rho_{\text {diel }}(\omega)=0$ при $|\omega| \leq E_{g}^{\text {sub }} / 2$, где $A-$ коэффициент с размерностью эB $^{-3 / 2}$ и нуль энергии помещен в центр запрещенной зоны подложки. Тогда при $|\omega| \leq E_{g}^{\text {sub }} / 2$ имеем $\Lambda_{\text {diel }}(\omega)=$ $=F_{-}(\omega)-F_{+}(\omega)$, где $F_{ \pm}(\omega)=\pi A V^{2} \sqrt{ \pm \omega+E_{g}^{\text {sub }} / 2}$ (см. подробнее [26]). Вопрос о переходе заряда в этом случае подробно проанализирован в [26]. Здесь мы этот вопрос не рассматриваем ${ }^{1}$.

Обращаясь к задаче о влиянии диэлектрической подложки на эффективную массу носителей в ГПС будем считать, что имеем дело с охватывающим гетеропереходом, изображенным на рис. $1, b$ работы [26]. Тогда, полагая $\bar{\varepsilon}=0$, получим $F(0)=\left(1+\pi A V^{2} /{\sqrt{E_{g}^{\mathrm{sub}} / 2}}^{-1}\right.$, так что $m^{*} / \widetilde{m}^{*}<1$. Более детальное рассмотрение этого вопроса преждевременно (см. сноску 1).

\footnotetext{
${ }^{1}$ Отказ от численных оценок перехода заряда между 2D $\mathrm{SiC}$ и диэлектрической подложкой связан с отсутствием какой-либо информации относительно электронного сродства и(или) работы выхода $2 \mathrm{D} \mathrm{SiC}$, что не позволяет определить взаиморасположение запрещенных зон $E_{g}$ и $E_{g}^{\text {sub }}$, весьма важное для оценок (см. [26]). Следует также отметить, что рассчитанные по таблицам атомных термов значения стандартного для приближения сильной связи параметра $\bar{\varepsilon}=\left(\varepsilon_{\mathrm{Si}}+\varepsilon_{\mathrm{C}}\right) / 2$ не отражают реального расположения зон относительно уровня вакуума, так как при этом не учитывается сдвиг атомных уровней в поле кристаллического потенциала. Поэтому в дальнейшей части работы вопрос о переходе заряда обсуждаться не будет.
} 


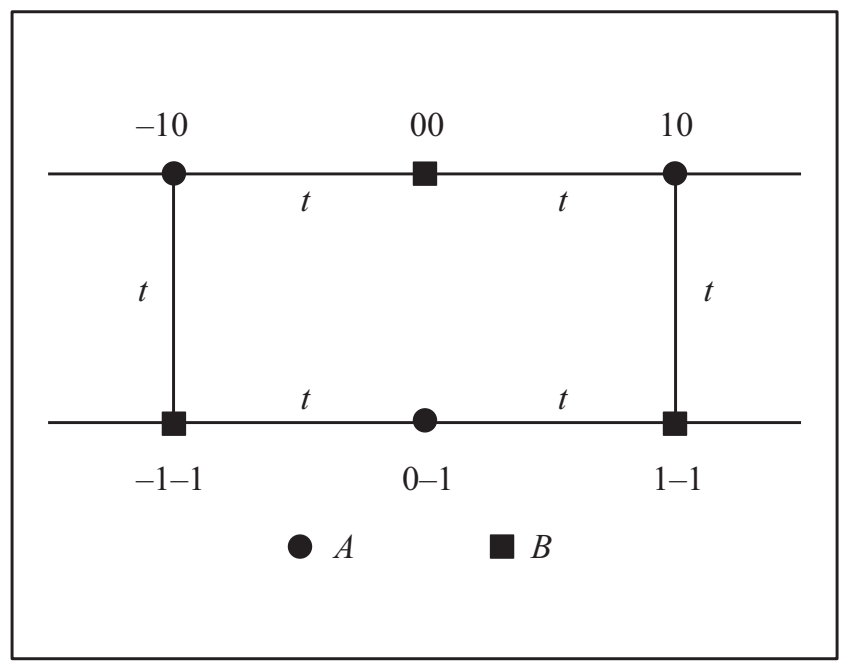

Pис. 1. Простейшая модель ZNR для $2 \mathrm{D}$ соединения $A B$ $(t-$ энергия перехода электрона между атомами $A$ и $B)$.

\section{3. Наноленты карбида кремния}

В работе [32] для эпитаксиального графена нами была предложена простейшая двухцепочечная модель наноленты с зигзагообразными краями (ZNR - zigzagtype nanoribbon). При этом использовалась решетка типа кирпичной стенки, топологически эквивалентная гексагональной решетке ГПС [33-35]. На рис. 1 аналогичная модель представлена для ZNR бинарного ГПС.

Сопоставим свободному изолированному атому $A(B)$ затравочную (bare) функцию Грина $g_{a(b)}(\omega)=$ $=\left(\omega-\varepsilon_{a(b)}+0^{+}\right)^{-1}$. Включая между атомами $A$ и $B$ взаимодействие $t$ (кинетический обмен), получим по аналогии с [32] следующие выражения для функций Грина внутренних (индекс 0) и внешних (индекс 1) атомов:

$$
\begin{gathered}
G_{0}^{A(B)}=g_{a(b)}\left(1-g_{a} g_{b} t^{2} \Phi-\frac{g_{a} g_{b} t^{2}}{1-g_{a} g_{b} t^{2} \Phi}\right)^{-1}, \\
G_{1}^{A(B)}=g_{a(b)}\left(1-\frac{g_{a} g_{b} t^{2} \Phi}{1-\frac{g_{a} g_{b} t^{2}}{1-g_{a} g_{b} t^{2} \Phi}}\right)^{-1},
\end{gathered}
$$

где $\Phi=4 \cos ^{2}(k a), k-$ волновой вектор для движения электрона вдоль наноленты, $a-$ расстояние между б.с. Полюса функций Грина (5) и (6), отвечающие корням уравнения

$$
\left(1-g_{a} g_{b} t^{2} \Phi\right)^{2}-g_{a} g_{b} t^{2}=0,
$$

определяют спектр системы вида

$$
\begin{gathered}
E_{l}(k)= \pm R_{ \pm}^{\prime}(k), \\
R_{ \pm}^{\prime}(k)=\left\{4 \Delta^{2}+t^{2}\left[(1+4 \Phi)^{1 / 2} \pm 1\right]^{2}\right\}^{1 / 2} / 2,
\end{gathered}
$$

где индекс $l$ нумерует зоны (здесь и далее принимаем $\bar{\varepsilon}=0)$. Условимся, что нумерация зон идет в сторону убывания энергии: $l=1$ соответствует знакам плюс перед $R^{\prime}(k)$ и в квадратной скобке, $l=2-$ плюсу перед $R^{\prime}(k)$ и минусу в квадратной скобке, $l=3$ - минусам в обоих случаях, $l=4-$ минусу перед $R^{\prime}(k)$ и плюсу в квадратной скобке. Для $2 \mathrm{D} \mathrm{SiC}$ зоны $E_{1}(k)=-E_{4}(k)$ и $E_{2}(k)=-E_{3}(k)$ представлены на рис. 2, $a$. При этом мы, для простоты, положили $\Delta / t=1$, что является промежуточным значением между $\Delta / t=0.80$ (таблицы Хермана-Скилманна [27]) и $\Delta / t=1.14$ (таблицы Манна [28]).

По аналогии с выражением (2) электронную эффективную массу для зоны $E_{1}(k)$ можно представить в
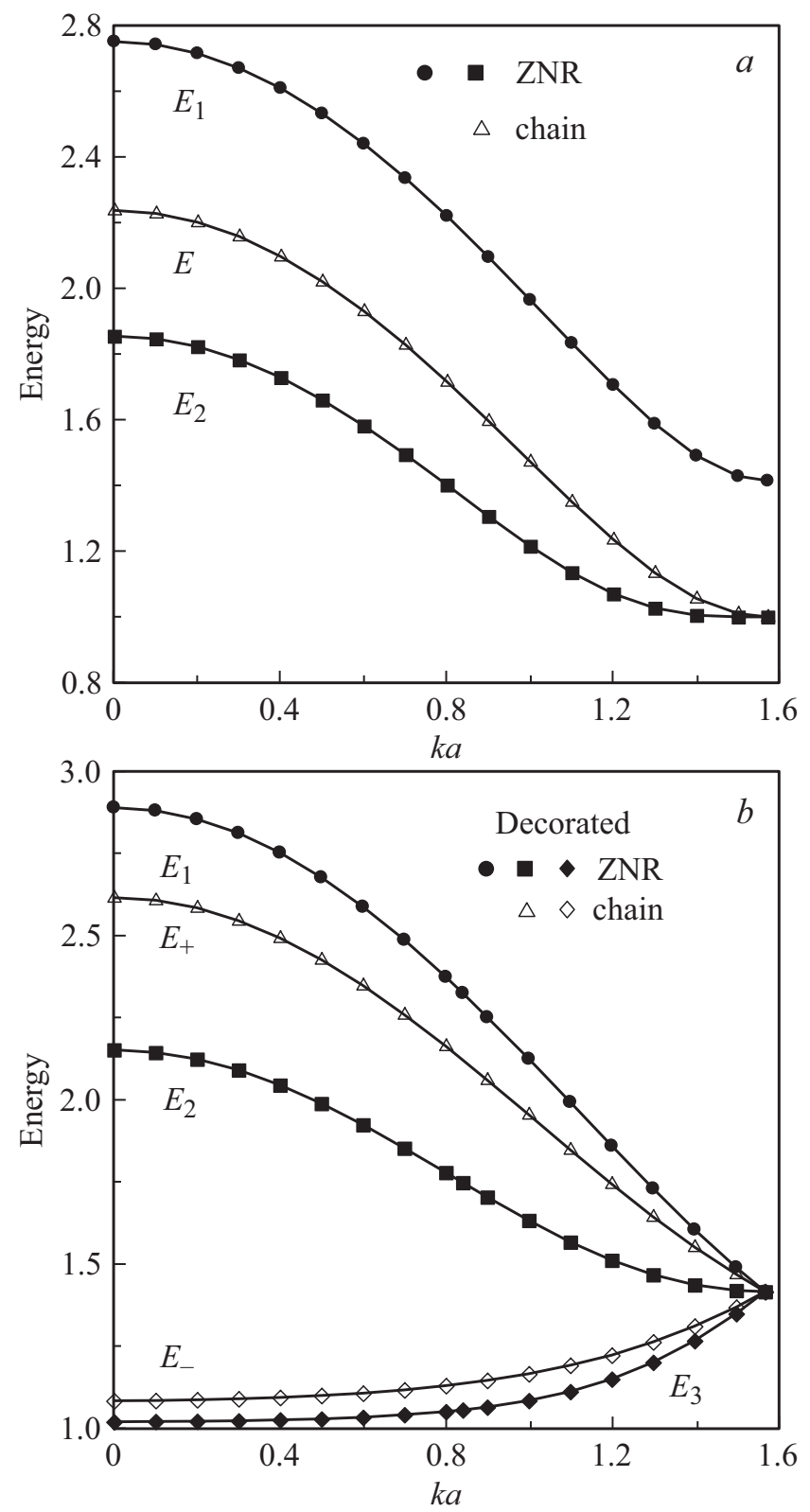

Рис. 2. Дисперсия зон недокорированных $(a)$ и декорированных $(b)$ ZNR (темные символы) и цепочки (светлые символы) $\mathrm{SiC}$ при $\Delta / t=1$ и $\bar{\varepsilon}=0$. Энергия измеряется в ед. $t^{-1}$. Изображены только зоны, отвечающие положительной энергии. 
Таблица 2. Результаты расчета ширины запрещенной зоны $E_{g}$ (в эВ), эффективной массы электрона $m^{*}=m_{e} / m_{0}$ и характерной скорости $c$ (в ед. $\left.10^{6} \mathrm{M} / \mathrm{c}\right)$ для наноструктур карбида кремния. Для листа приведены значения $E_{g}$ и $m^{*}$, рассчитанные с использованием таблиц Хермана-Скиллмана и Манна; в остальных случаях принято $\Delta=t=1.53$ эВ

\begin{tabular}{|c|c|c|c|c|c|}
\hline Структура & Лист & ZNR & $\begin{array}{c}\text { Декорированная } \\
\text { ZNR }\end{array}$ & Цепочка & $\begin{array}{c}\text { Декорированная } \\
\text { цепочка }\end{array}$ \\
\hline$E_{g}, \ni \mathrm{B}$ & $\begin{array}{l}2.45 \\
3.48\end{array}$ & 3.06 & 3.12 & 3.06 & 3.31 \\
\hline $\begin{array}{c}m^{*} \\
k=\pi / 2 a\end{array}$ & $\begin{array}{l}0.57 \\
0.81\end{array}$ & $\begin{array}{c}0.28 \\
-\end{array}$ & 0.56 & 0.40 & - \\
\hline $\begin{array}{l}m^{*} \\
k=0\end{array}$ & - & - & 58 & - & 37 \\
\hline $\begin{array}{c}c, \\
k=\pi / 2 a\end{array}$ & $\begin{array}{l}- \\
-\end{array}$ & $\begin{array}{l}- \\
-\end{array}$ & 0.41 & $\begin{array}{l}- \\
-\end{array}$ & 0.29 \\
\hline
\end{tabular}

виде $m_{1}^{-1}=c_{1}^{2} / \Delta$, где $c_{1}=2 \sqrt{2} a t / \hbar$ и $\Delta_{1}=\sqrt{\Delta^{2}+t^{2}}$. Для $2 \mathrm{D} \mathrm{SiC}$ при $\Delta / t=1$ получаем $m_{1}^{*}=0.28$. Для зоны $E_{2}(k)$ вблизи границы зоны Бриллюэна ZNR при малых $q^{\prime}=(\pi / 2 a)-k$ имеем $R_{-}^{\prime}\left(q^{\prime}\right) \approx \Delta+8 t^{2}\left(q^{\prime} a\right)^{4} / \Delta$. Дисперсия такого вида не позволяет использовать стандартное определение эффективной массы. Здесь мы имеем дело с характерным для ZNR плоским участком зоны (см. рис. 2, a). Отметим в связи с этим полученные в [22] ближние к щели зоны с характерным уплощением в области границы зоны Бриллюэна (см. рис. 3 в [22]). Для примера: в случае графеновой ZNR из выражения (8) при $\Delta=0$ получаем $E_{\mathrm{Gr}}(k)= \pm t \Phi(k)$, где нуль энергии совмещен с точкой Дирака. Тогда эффективная масса электрона $m_{\mathrm{Gr}}^{-1}=c_{\mathrm{Gr}}^{2} / t_{\mathrm{Gr}}$, где $c_{\mathrm{Gr}}=2 \sqrt{2} a_{\mathrm{Gr}} t_{\mathrm{Gr}} / \hbar$. Так как $a_{\mathrm{Gr}}=1.42 \AA$ и $t_{\mathrm{Gr}}=2.38$ эВ (рассчитано по Харрисону), получаем $m_{\mathrm{Gr}}^{*}=0.20$.

Усложним теперь модель путем специфического декорирования зубцов ZNR собственными атомами, а именно присоединим к атомам $B$ типа 00 и атомам $A$ типа 0-1 соответственно атомы $A$ и $B$, полагая, что вновь образованные связи имеют ту же длину $a$ и, следовательно, отвечают взаимодействию $t$. Отметим, что такая нанолента уже не является ZNR (равно как и ANR (armchair-type nanoribbon)), поскольку внешние атомы имеют только одного б. с.

Воспользовавшись результатами работы [32], легко показать, что закон дисперсии определяется теперь уравнением вида

$$
\left[1-g_{a} g_{b} t^{2}(1+\Phi)\right]^{2}-g_{a} g_{b} t^{2}\left(1-g_{a} g_{b} t^{2}\right)^{2}=0 .
$$

Графики зависимостей $E_{t}(k)$ при $l=1,2,3$ для положительных энергий изображены на рис. $2, b$ (темные символы). Обращает на себя внимание резкое отличие этих зависимостей от графиков, представленных для недекорированной ZNR (рис. 2,a). Во-первых, декорирование ведет к появлению зон $E_{3}=-E_{4}$, имеющих минимумы в центре зоны Бриллюэна наноленты. Учитывая, что в пределе $k \rightarrow 0$ справедливо неравенство $g_{a} g_{b} t^{2} \gg 1$, из (9) получим

$$
E_{3}(k) \approx \sqrt{\Delta^{2}+t^{2} /\left[2+(1+\Phi)^{2}\right]} .
$$

Так как $t=\Delta$ и $\Phi(0)=4$, имеем $m_{3}^{*} \approx 58$. Таким образом, можно считать, что в окрестности центра зоны Бриллюэна $E_{3}(k)=$ const.

Во-вторых, вблизи границы зоны Бриллюэна в первом приближении по $\Phi \ll 1$ для зон, отвечающих положительной энергии, имеем дисперсии вида

$$
\begin{aligned}
& E_{2}\left(q^{\prime}\right) \approx \sqrt{\Delta^{2}+t^{2}}\left(1+\frac{2\left(q^{\prime} a\right)^{2} t^{2}}{\Delta^{2}+t^{2}}\right), \\
& E_{l}\left(q^{\prime}\right) \approx \sqrt{\Delta^{2}+t^{2}}\left(1 \pm \frac{\sqrt{2} q^{\prime} a t^{2}}{\Delta^{2}+t^{2}}\right),
\end{aligned}
$$

где знак плюс относится к зоне с $l=1$, а знак минус - к зоне с $l=3$. Таким образом, зоне $E_{2}$ отвечает электронная эффективная масса $m_{2}^{-1} \approx c_{2}^{2} / \sqrt{\Delta^{2}+t^{2}}$, где $c_{2}=2 a t / \hbar$, откуда получаем $m_{2}^{*}=0.56$. Зоны $E_{1}$ и $E_{3}$ с линейным спектром характеризуются скоростями $c_{1,3} \approx \pm \sqrt{2} a t^{2} / \hbar \sqrt{\Delta^{2}+t^{2}}= \pm 0.41 \cdot 10^{6} \mathrm{M} / \mathrm{c}$. При $k=\pi / 2 a$ имеем трехкратно вырожденные значения $E_{l}= \pm \sqrt{\Delta^{2}+t^{2}}$, где знак плюс соответствует зонам $1,2,3$, знак минус - зонам $4,5,6$. Отметим, что при декорировании ZNR ширина запрещенной зоны равна $E_{g}=2 \Delta(1+\vartheta)$, где $\vartheta \ll 1$ (см. табл. 2 и рис. $\left.2, b\right)$.

Влияние металлической и диэлектрической подложек на скорости Ферми и эффективные массы носителей тока в ZNR и декорированной ZNR такое же, как и в случае бесконечного листа ГПС (разд. 2). Дело в том, что функция сдвига $\Lambda(\omega)$, входящая в соотношения (4), определяется как

$$
\Lambda(\omega)=V^{2} \int_{-\infty}^{\infty} \frac{\rho_{\mathrm{sub}\left(\omega^{\prime}\right) d \omega^{\prime}}}{\omega-\omega^{\prime}},
$$

где $\rho_{\text {sub }}(\omega)-$ плотность состояний подложки. Таким образом, если для различных эпитаксиальных наноструктур задавать одну и ту же функцию $\rho_{\text {sub }}(\omega)$, то значения 


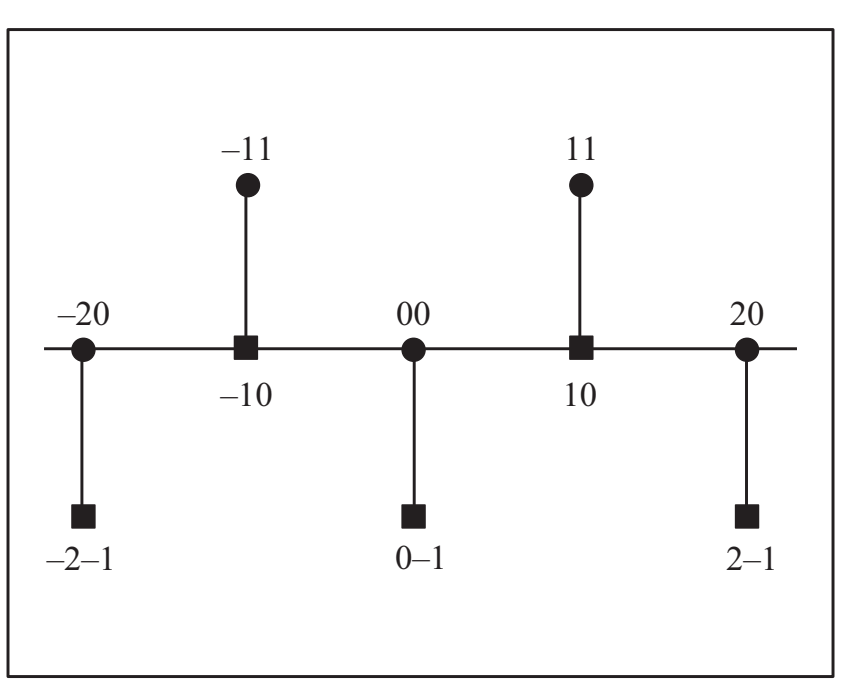

Рис. 3. Декорированная цепочка для $2 \mathrm{D}$ соединения $A B$.

функции $F(\omega)$ (4) будут качественно одинаковы. Количественные отличия будут связаны лишь с величиной матричного элемента взаимодействия наноструктуры с подложкой $V$. Приведенные рассуждения относятся и к цепочечным структурам.

\section{4. Цепочки карбида кремния}

Для цепочки (одномерная структура) бинарного соединения $A B$ функции Грина равны $G^{A(B)}=g_{a(b)}$ $\times\left(1-g_{a} g_{b} t^{2} \Phi\right)^{-1}$, так что закон дисперсии имеет стандартный вид, присущий двухатомной цепочке (см., например, [30]):

$$
E(k)= \pm \sqrt{\Delta^{2}+t^{2} \Phi}
$$

(см. кривую, изображенную на рис. 2, $a$ светлыми треугольниками). Таким образом, электронная эффективная масса есть $m^{-1}=c^{2} / \Delta$, где $c=2 a t / \hbar$. Отсюда для цепочки $\mathrm{SiC}$ имеем $m^{*}=0.40$, где мы использовали (для непосредственного сравнения с предыдущими результатами) те же значения параметров $a$ и $t$, что и выше. Отметим, что характеристики низкоразмерных структур $\mathrm{SiC}$ рассчитывались DFT-методом (density functional theory) в [23]. В частности, было получены значения $a$, равные $1.786 \AA$ для 2D (что на $1 \%$ больше принятой нами величины) и $1.649 \AA$ для $1 \mathrm{D}$ (что на 7\% меньше использованного нами значения). Здесь, однако, эти поправки игнорируем, так как в настоящей работе ставим целью получить лишь полуколичественные оценки.

Для декорированной цепочки (квазиодномерная структура), изображенной на рис. 3, найдем функции Грина вида

$$
G_{0}^{A(B)}=g_{a(b)}\left(1-g_{a} g_{b} t^{2}-\frac{g_{a} g_{b} t^{2} \Phi}{1-g_{a} g_{b} t^{2}}\right)^{-1},
$$

$$
G_{1}^{A(B)}=g_{a(b)}\left(1-\frac{g_{a} g_{b} t^{2}}{1-\frac{g_{a} g_{b} t^{2} \Phi}{1-g_{a} g_{b} t^{2}}}\right)^{-1},
$$

где индексы 0 и 1 относятся к атомам исходной цепочки (типа $n_{0}$ ) и декорирующим атомам соответственно. Полюсами функций Грина (15) и (16) являются корни уравнения

$$
\left(1-g_{a} g_{b} t^{2}\right)^{2}-g_{a} g_{b} t^{2} \Phi=0 .
$$

Отсюда для области положительных энергий находим закон дисперсии

$$
E_{ \pm}(k)=\sqrt{\Delta^{2}+\frac{1}{2} t^{2}(2+\Phi \pm \sqrt{\Phi(4+\Phi)})}
$$

Графики зависимостей $E_{ \pm}(k)$ представлены на рис. $2, b$. Вблизи границы зоны Бриллюэна получаем

$$
E\left(q^{\prime}\right) \approx \pm\left(\Delta^{2}+t^{2}\right)^{1 / 2}\left(1 \pm \frac{q^{\prime} a t^{2}}{\Delta^{2}+t^{2}}\right)
$$

Имеем, таким образом, спектр со щелью, но с линейной дисперсией, характеризуемой скоростью $c_{+} \approx a t^{2} / \hbar \sqrt{\Delta^{2}-t^{2}}=0.29 \cdot 10^{6} \mathrm{M} / \mathrm{c}$. (Для сравнения отметим, что для бесконечного листа графена $\left.v_{\mathrm{F}}(\mathrm{Gr})=3 a_{\mathrm{Gr}} t_{\mathrm{Gr}} / 2 \hbar=0.77 \cdot 10^{6} \mathrm{M} / \mathrm{c}\right)$. Для центра зоны Бриллюэна из (19) имеем $m_{-}^{-1}=c_{-}^{2} / \Delta$, где $c_{-} \approx a t \sqrt{(3 \sqrt{2} / 4)-1} / \hbar$ и $\Delta_{-} \approx \sqrt{\Delta^{2}+t^{2}}$, так что $m_{-}^{*} \approx 37$. Таким образом, как и в случае декорированной наноленты, получаем аномально высокое значение эффективной массы электрона, больше напоминающее эффективные массы поляронов [36,37].

\section{5. Заключение}

Итак, в настоящей работе с использованием простых структурных моделей мы получили аналитические выражения для электронных спектров находящихся в свободном состоянии нанолент и цепочек $\mathrm{SiC}$, что позволило впервые, насколько известно автору, оценить эффективные массы носителей $m^{*}$ в случае квадратичной дисперсии и характерные скорости $c$ в случае линейной дисперсии. Для удобства полученные результаты сведены в табл. 2. Продемонстрировано, что декорирование двухцепочечной наноленты и одномерной структуры качественно меняет электронный спектр: в частности, происходит „смещение“ запрещенной зоны от границы зоны Бриллюэна в ее центр. При этом резко меняются и значения зонных характеристик. Для квазисвободных наноструктур кратко обсуждается вопрос о влиянии подложки (металлической и диэлектрической) на значения $m^{*}$ и $c$. Для более тщательного анализа требуются дополнительные экспериментальные данные. Дело в том, что даже при расчетах из первых принципов имеются существенные расхождения как относительно 
значений $E_{g}$, так и расположения щели в зоне Бриллюэна (см. табл. 1).

В заключение стоит отметить, что в настоящее время уже имеются 2D SiC-структуры, сформированные на кремнии [38] и графене [39]. Особое место занимают композиты $2 \mathrm{D} \mathrm{SiC} / \mathrm{SiC}[40,41]$.

\section{Конфликт интересов}

Автор заявляет об отсуствии конфликта интересов.

\section{Список литературы}

[1] А.К. Гейм. УФН, 181, 1284 (2011).

[2] К.С. Новосёлов. УФН, 181, 1299 (2011).

[3] A.H. Castro Neto, F. Guinea, N.M.R. Peres, K.S. Novoselov, A.K. Geim. Rev. Mod. Phys., 81, 109 (2009).

[4] M.I. Katsnelson. Graphene. Carbon in Two Dimensions (UK, Cambridge University Press, 2012).

[5] Graphene Nanoelectronics. Metrology, Synthesis, Properties and Applications, ed. by H. Raza. (Springer Verlag, BerlinHeidelberg, 2012).

[6] П.Б. Сорокин, Л.А. Чернозатонский. УФН, 183, 113 (2013).

[7] A.K. Geim, I.V. Grigorieva. Nature, 499, 419 (2013).

[8] M. Xu, T. Liang, M. Shi, H. Chen. Chem. Rev., 113, 3766 (2013).

[9] G. Mukhopadhyay, H. Behera. World J. Engin., 10, 39 (2013).

[10] C.-J. Tong, H. Zhang, Y.-N. Zhang, H. Liu, L.-M. Liu. J. Mater. Chem. A, 2, 17971 (2014).

[11] И.В. Антонова. ФТП, 50, 67 (2016).

[12] R. Roldán, L. Chirolli, E. Prada, J.A. Silva-Guillén, P. San Josea, F. Guinea. Chem. Soc. Rev., 46, 4387 (2017).

[13] H. Şahin, S. Cahangirov, M. Topsakal, E. Bekaroglu, E. Akturk, R.T. Senger, S. Ciraci. Phys. Rev. B, 80, 155453 (2009).

[14] T. Suzuki, Y. Yokomizo. Physica E, 42, 2820 (2010).

[15] S. Wang. J. Phys. Soc. Jpn., 79, 064602 (2010).

[16] H.L. Zhuang, A.K. Singh, R.G. Hennig. Phys. Rev. B, 87, 165415 (2013).

[17] A.K. Singh, H.L. Zhuang, R.G. Hennig. Phys. Rev. B, 89, 245431 (2014).

[18] D. Kecik, A. Onen, M. Konuk, E. Gürbüz, F. Ersan, S. Cahangirov, E. Aktürk, E. Durgun, S. Ciraci. Appl. Phys. Rev., 5, 011105 (2018).

[19] Silicon carbide: recent major advances, W.J. Choyke, H. Matsunami, G. Pensl (eds) (Springer Verlag, Berlin-HeidelbergN.Y., 2004).

[20] A.A. Lebedev. Semicond. Sci. Technol., 21, R17 (2006).

[21] B. Baumeier, P. Krüger, J. Pollmann. Phys. Rev. B, 76, 085407 (2007).

[22] L. Sun, Y. Li, Z. Li, Q. Li, Z. Zhou, Z. Chen, J. Yang, J.G. Hou. J. Chem. Phys., 129, 174114 (2008).

[23] E. Bekaroglu, M. Topsakal, S. Cahangirov, S. Ciraci. Phys. Rev. B, 81, 075433 (2010).

[24] T. Susi, V. Skákalová, A. Mittelberger, P. Kotrusz, M. Hulman, T.J. Pennycook, C. Mangler, J. Kotakoski, J.C. Meyer. Sci. Rep., 7, 4399 (2017).

[25] С.Ю. Давыдов. ФТТ, 58, 779 (2016).

[26] С.Ю. Давыдов. ФТТ, 58, 1182 (2016).
[27] У. Харрисон. Электронная структура и свойства твердых тел (М., Мир, 1983) т. 1.

[28] W.A. Harrison. Phys. Rev. B, 31, 2121 (1985).

[29] В.И. Гавриленко, А.М. Грехов, Д.В. Корбутяк, В.Г. Литовченко. Оптические свойства полупроводников. Справочник. (Киев, Наук. думка, 1987).

[30] С.Ю. Давыдов, А.А. Лебедев, О.В. Посредник. Элементарное введение в теорию наносистем (СПб., Лань, 2014).

[31] С.Ю. Давыдов. Письма ЖТФ, 45 (13), 14 (2019).

[32] С.Ю. Давыдов. ФТТ, 61, 610 (2019).

[33] K. Wakabayashi, M. Fujita, H. Ajiki, M. Sigrist. Phys. Rev. B, 59, 8271 (1999).

[34] J. Cserti. Am. J. Phys., 68, 896 (2000).

[35] G. Jose, R. Malla, V. Srinivasan, A. Sharma, S. Gangadharaiah. arXiv: $1711.08204 \mathrm{v} .1$

[36] G. Ganbold, G.V. Efimov. J. Phys.: Condens. Matter, 10, 4845 (1998).

[37] A.H. Romero, D.W. Brown, K. Lindenberg. Phys. Rev. B, 59, 13728 (1999).

[38] C.W. Petza, D. Yang, J. Levy, J.A. Floro. J. Mater. Res., 28, 261 (2013).

[39] S. Lin, S. Zhang, X. Li, W. Xu, X. Pi, X. Liu, F. Wang, H. Wu, H. Chen. J. Chem. Phys. C, 119, 19772 (2015).

[40] A. Mouchtachi, R. El Guerjouma, J.C. Baboux, D. Rouby, D. Bouami. J. Phys. D: Appl. Phys., 37, 3323 (2004).

[41] X. Liu, X. Shen, L. Gong, P.Li. Chin. J. Aeronautics, 31, 117 (2018).

Редактор Г.А. Оганесян

\section{Silicon carbide low-dimensional structures: analytical estimates of the electron spectrum characteristics}

\section{S.Yu. Davydov}

loffe Institute,

194021 St. Petersburg, Russia

Abstract By the use of Green's functions method within the tight-binding approximation the electronic structure for the silicon carbide sheet, nunoribbons and chains are considered. Analytical expressions for the gap values, effective masses and characteristic velocities for the charge carriers are obtained. The influence of metallic and dielectric substrates on the band characteristics are discussed. 